\title{
Antitumoral Efficacy of Two Turmeric Extracts According to Different Extraction Methods in Hepatocellular Carcinoma Cell Lines
}

\author{
Marzia Deserti ${ }^{1,2 \#}$, Simona Tavolari ${ }^{1,2 \#}$, Marina Naldi ${ }^{2,3}$, Giorgio Frega ${ }^{1}$ and Giovanni Brandi $i^{1,2^{*}}$ \\ ${ }^{1}$ Department of Experimental, Diagnostic and Specialty Medicine, S. Orsola-Malpighi University Hospital, Bologna, Italy \\ ${ }^{2}$ Center for Applied Biomedical Research, S. Orsola-Malpighi University Hospital, Bologna, Italy \\ ${ }^{3}$ Department of Pharmacy and Biotechnology, University of Bologna, Bologna, Italy \\ \#Contributed equally
}

*Corresponding author: Prof. Giovanni Brandi, MD, PhD, Department of Experimental, Diagnostic and Specialty Medicine, S Orsola-Malpighi University Hospital, via Massarenti 9, 40138, Bologna, Italy, Tel: +390512143838; Fax: +390512144037; E-mail: giovanni.brandi@unibo.it

Received date: October 31, 2018; Accepted date: November 19, 2018; Published date: November 26, 2018

Copyright: (c) 2018 Deserti M, et al. This is an open-access article distributed under the terms of the Creative Commons Attribution License, which permits unrestricted use, distribution, and reproduction in any medium, provided the original author and source are credited.

\begin{abstract}
Curcuminoids, bioactive molecules contained in turmeric, have been reported to exert anticancer effects in several human cancers, including hepatocellular carcinoma (HCC). However, the extraction method can significantly affect the structural characteristics of curcuminoids and their biological properties. On this basis, in the present study we investigated the content of curcuminoids and the anticancer activity of two turmeric powders extracted according two different methodologies: solvent extraction with ethyl acetate vs an ancient Indian extraction method of boiling of rhizomes in water followed by dehydration at the sun. Results obtained showed that extraction with ethyl acetate resulted in a significant recovery of curcuminoids and anticancer activity both in terms of cell cytotoxicity and migration/invasiveness inhibition in HCC cell lines, compared to common Indian practice. Overall these findings suggest that turmeric powders could have different efficacy, depending on the extraction method. This aspect should be taken into account when choosing the best product to be employed in the prevention and treatment of human diseases, including cancer.
\end{abstract}

Keywords: Turmeric; Extraction; Curcuminoids; Hepatocellular carcinoma; Cytotoxicity; Cancer

\section{Introduction}

Turmeric, the dried ground rhizome of Curcuma longa L., is a spice cultivated in tropical and subtropical regions, especially in India. Traditionally, it has been employed in Ayurvedic medicine in light of its anti-inflammatory properties. However, several in vitro and in vivo studies have clearly shown that turmeric extracts harbor additional biological properties, including powerful antioxidant, antibacterial, anti-viral and antitumoral activities [1]. This large biological activity sets turmeric extracts as promising molecules for the prevention and treatment of many human diseases, including cancer [2]. The biological properties of turmeric extracts are known to be mainly linked to the content of curcuminoids, bioactive molecules including curcumin (the principal constituent), demethoxycurcumin and bisdemethoxycurcumin (Figure 1A). Due to the beneficial health effects, curcumin and their derivatives have been the object of extensive investigation in the field of biology, medicine and pharmacology. However, the potential application of curcuminoids as bioactive molecules is limited by their low solubility and poor stability [3]. Currently different conventional methodologies are employed to extract curcuminoids from turmeric, including extraction with solvents [4-7]. According to the international authorities that monitor the development of food additives, a limited number of solvents is allowed for preparation of curcuminoid-based products, in particular ethyl acetate. Besides conventional methodologies, a traditional Indian practice is the boiling of rhizomes in water followed by dehydration at the sun. This practice, along with drying conditions, has been reported to influence the level of curcuminoids in the rhizomes [8]. Since the structural characteristics of curcuminoids and their biological properties can be significantly affected by extraction method [9], in the present study we compared the efficacy of conventional solvent extraction with ethyl acetate vs the common Indian extraction in neutral aqueous solution. The antitumoral properties of the turmeric powders obtained according to these two different methodologies was then investigated on HepG2 and Huh-7 human hepatocellular carcinoma (HCC) cell lines.

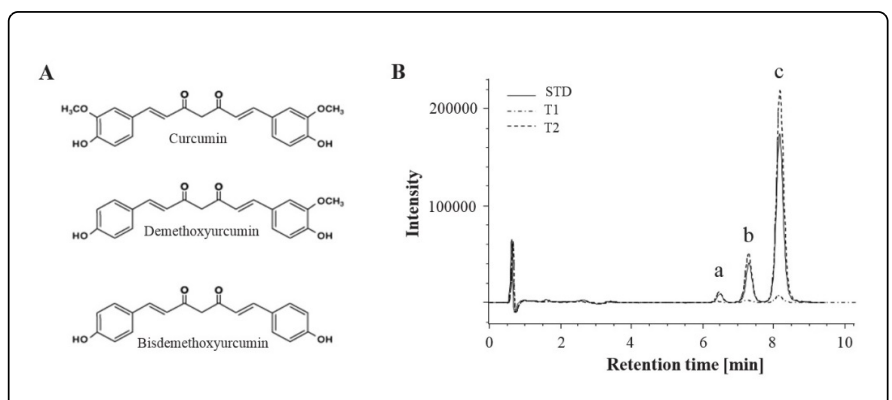

Figure 1: A) Chemical structure of curcuminoids; B) Chromatogram of curcuminoids at $405 \mathrm{~nm}$. For each turmeric powder (STD, T1 and T2), three different peaks are reported: bisdemethoxycurcumin (a), demethoxycurcumin (b) and curcumin (c). STD: standard. 


\section{Materials and Methods}

\section{Cell culture}

The human HCC cell lines Huh-7 and HepG2 were kindly provided by Dr. Catia Giovanni (Center for Applied Biomedical Research, S. Orsola-Malpighi University Hospital, Bologna, Italy) and maintained in Dulbecco's modified Eagle's Medium with g/L glucose (Euroclone, Milan, Italy), supplemented with $10 \%(\mathrm{v} / \mathrm{v})$ heat-inactivated FBS (Euro clone), $2 \mathrm{mM}$ L-glutamine, $100 \mathrm{U} / \mathrm{ml}$ penicillin and $100 \mu \mathrm{g} / \mathrm{ml}$ streptomycin (Sigma-Aldrich, St. Louis, MO, USA) in an atmosphere of $5 \% \mathrm{CO}_{2}$ at $37^{\circ} \mathrm{C}$.

\section{Turmeric powders}

Turmeric powder 1 (T1) was purchased from an Ayurvedic Laboratory in Nepal. Briefly, raw turmeric rhizomes cultivated in Nepal at $2000 \mathrm{mt}$ were harvested in June, cleaned to remove hairy roots and boiled in water for 45 minutes until the rhizomes became soft. Water was then drained, and the turmeric's were sun dried for 10-15 days until they became dry and hard. The dried product was cleaned and polished and finally ground by hand with stone mills. Turmeric powder 2 (T2) was obtained from an herbalist's shop located in Bologna (Italy). It was produced with turmeric rhizome grown in India and curcuminoids extraction was carried out according to conventional ethyl acetate method [10]. A turmeric powder containing a mixture of curcuminoids ( $99 \%$ purity, Sigma-Aldrich, St. Louis, MO, USA) was used as reference standard (STD). All turmeric powders were diluted into DMSO to obtain the final concentration before each experiment.

\section{Curcuminoids LC-UV analysis}

STD, T1 and T2 turmeric solutions $(1 \mathrm{mg} / \mathrm{mL}$, in methanol) were diluted 40 folds with water-acetonitrile-FA (99:1:0.1, v/v/v) mixture. $\mathrm{T} 1$ resulted to be not completely soluble in methanol; for this reason, its solution was filtered with a $0.22 \mu \mathrm{m}$ filter (Merck KGaA, Darmstadt, Germany). In details, curcumin was determined using a Jasco PU-1585HPLC system (Jasco Corporation, Tokyo, Japan) equipped with a Rheodyne 7281 injection valve ( $20 \mu \mathrm{L}$ sample loop). The detection was performed with a Jasco UV-1575 detector set at $405 \mathrm{~nm}$. The analysis was performed using an Agilent extend C18 chromatographic column $(3.5 \mu \mathrm{m} ; 2.1 \times 100 \mathrm{~mm})$. The analyses were performed in isocratic mode using a mobile phase composed of wateracetonitrile-FA $(63: 37: 0.1, \mathrm{v} / \mathrm{v} / \mathrm{v})$. The injection volume was $20 \mu \mathrm{L}$ and the flow rate was $0.5 \mathrm{~mL} / \mathrm{min}$. We expected to obtain three different peaks corresponding to the $C$. longa curcuminoids: bisdemethoxycurcumin (A), demethoxycurcumin (B) and curcumin (C). The peak identity was attributed both by the characteristic relative abundance of each curcuminoid, both by the elution order in a reverse phase approach, as reported in literature $[11,12]$.

\section{Cell viability assay}

Cell viability experiments were carried out by 3-[4,5dimethylthiazol-2-yl]-2,5-diphenyltetrazolium bromide (MTT) assay. Briefly, Huh-7 and HepG2 cells $\left(5 \times 10^{3}\right.$ cells/well) were plated in a 96well plate in triplicate and allowed to adhere for $24 \mathrm{hrs}$. Cells were then treated with increasing concentrations $(10,25$ and $50 \mu \mathrm{M})$ of DMSO or STD, T1 and T2 turmeric powders for 24, 48 and $72 \mathrm{hrs}$. At the end of incubation, MTT was added to each well and cells incubated at $37^{\circ} \mathrm{C}$ for $4 \mathrm{~h}$. Formazan crystals were then dissolved by DMSO addition.
Absorbance was then measured at $570 \mathrm{~nm}$ in a 96-well spectrophotometric microplates reader (Bio-Rad, Hercules, CA, USA).

\section{Cell cycle analysis by flow cytometry}

For the analysis of DNA content, Huh-7 and HepG2 cells were seeded on a six-well plate at the density of $4 \times 10^{5}$ cells/well and treated with DMSO or STD, T1 and T2 turmeric powders at $50 \mu \mathrm{M}$ concentration. At different intervals $(24,48$, and $72 \mathrm{hrs})$, cells were harvested by trypsinization, washed with cold phosphate-buffered saline (PBS) and incubated at $4^{\circ} \mathrm{C}$ for $16 \mathrm{~h}$ in the DNA-staining solution (containing $0.1 \%$ Triton $\mathrm{X}-100,0.1 \%$ sodium citrate and 50 $\mu \mathrm{g} / \mathrm{ml}$ propidium iodide). At the end of incubation, cells were harvested, washed in PBS. DNA content analysis was performed by FACSAria $^{\text {tw }}$ cell sorter (Becton Dickinson, Franklin Lakes, NJ).

\section{In vitro wound healing (scratch) assay}

Cell motility assay was assessed by wound healing (scratch) assay. Briefly Huh-7 and HepG2 $\left(4 \times 10^{5}\right.$ cells/well $)$ were seeded in 6-well plates and allowed to attach to the surface under standard incubation conditions. After $24 \mathrm{hrs}$, the confluent cell monolayer was scratched in a straight line using a $200-\mu \mathrm{l}$ sterile plastic pipette tip. The cells were then carefully rinsed with culture medium to remove free-floating cells and debris. Then $50 \mu \mathrm{M}$ DMSO or STD, T1 and T2 turmeric powders were added to each well and the effect on wound healing was monitored. Scratch zones representative for each cell line were photographed immediately and after $48 \mathrm{hrs}$ of treatment with an Olympus CK $\times 41$ inverted microscope, equipped with an Olympus C5060-ADU camera (Olympus, Milan, Italy).

\section{In vitro matrigel invasion assay}

Huh-7 and HepG2 invasive potential was assessed by Matrigel invasion assay. Briefly, $12 \mu \mathrm{m}$ polyvinylpyrrolidone-free polycarbonate filters (Millipore Co, Cork, Ireland) were coated with growth factorreduced Matrigel (BD Biosciences, Milan, Italy). Complete medium was placed in the lower chamber as chemo-attractant. Huh-7 and HepG2 $\left(5 \times 10^{5}\right)$ cells, previously treated for $48 \mathrm{hrs}$ with DMSO or STD, T1 and T2 turmeric powders at $50 \mu \mathrm{M}$, were seeded in the upper chamber and incubated for 6 hours at $37^{\circ} \mathrm{C}$ in a humidified $5 \% \mathrm{CO}_{2}$. At the end of incubation, non-invading cells were removed from the upper surface of the filters, and invading cells in the lower surface were fixed for $1 \mathrm{~min}$ in ethanol $95 \%$ and stained for $10 \mathrm{~min}$ with $0.5 \% \mathrm{wt} / \mathrm{vol}$ toluidine blue. For each sample, 4 random optical fields at x 200 of total magnification were analyzed.

\section{Statistical evaluation}

Results were expressed as mean or percentage mean \pm SD and reproducibility was confirmed in three separate experiments. Differences between groups were analyzed using Student $t$ test. Data were analyzed by Prism 5.0 program and a $\mathrm{P}$ value $<0.05$ was considered to be statistically significant.

\section{Results}

\section{Evaluation of the curcuminoids content in T1 and T2 turmeric powders}

We firstly evaluated the curcuminoids content in T1 and T2 turmeric powders; STD turmeric powder was used as reference. For 
Citation: Deserti M, Tavolari S, Naldi M, Frega G, Brandi G (2018) Antitumoral Efficacy of Two Turmeric Extracts According to Different Extraction Methods in Hepatocellular Carcinoma Cell Lines. Med Aromat Plants (Los Angeles) 7: 324. doi:10.4172/2167-0412.1000324

Page 3 of 4

this purpose, a reverse-phase HPLC-UV assay was carried out using an isocratic approach. As described above, for sample preparation $1 \mathrm{mg}$ of each formulation was solubilized in $1 \mathrm{~mL}$ of methanol. STD and T2 powders resulted completely soluble in methanol; conversely $\mathrm{T} 1$ was not completely soluble and its filtration resulted necessary. Therefore, for T1, a lower purity in terms of curcuminoids content was expected. The overlaid chromatograms obtained with the LC-UV analyses are reported in Figure 1B. In all samples, three distinct peaks (a, b and c), corresponding to the different $C$. longa curcuminoids, were observed; in particular, T2 turmeric powder contained the highest quantity of curcumin. Assuming the peak area of T2 as $100 \%$ (Benchmark), the peak area and, consequently, the curcumin content of STD was equivalent to the $95 \%$, while that of $\mathrm{T} 1$ reached only the $5 \%$.

\section{T2, but not T1, inhibits migration and invasive potential of HepG2 and HuH-7 cell lines}

As already reported, curcuminoids have a strong anti-proliferative effect on cancer cells [13]. Therefore we tested the effect on HepG2 and $\mathrm{HuH}-7$ cell viability of increasing concentrations $(0-10-25-50 \mu \mathrm{M})$ of STD, T1 and T2 turmeric powders after 24, 48 and $72 \mathrm{hrs}$ of treatment. As reported in Figure $2 \mathrm{~A}$ and $2 \mathrm{~B}$, a significant time-and dosedependent decrease of cell viability was observed in both cell lines treated with T2 powder, reaching about the $90 \%$ in $\mathrm{HuH}-7$ cells and $80 \%$ in HepG2 cells after 72 hrs of treatment at the dose of $50 \mu \mathrm{M}$. Conversely, treatment for $72 \mathrm{hrs}$ with T1 powder, also at high concentrations, did not exert any significant effect on HepG2 and HuH-7 cell viability. Next, we investigated the effect of T1 and T2 turmeric powders on HepG2 and HuH-7 cell cycle. As shown in Figure $3 \mathrm{~A}$, treatment with T2 (but not with T1) powder at the dose of $50 \mu \mathrm{M}$ induced a time-dependent increase in the sub-G0 phase, which represents the fraction of fragmented apoptotic cells. In particular after $72 \mathrm{hrs}$ of treatment, we observed an induction of apoptosis reaching about $90 \%$ of apoptotic cells in HuH-7 and 50\% in HepG2 cell lines (Figure 3B). No significant changes in cell cycle and apoptosis were instead observed for T1 turmeric powder (Figure 2).
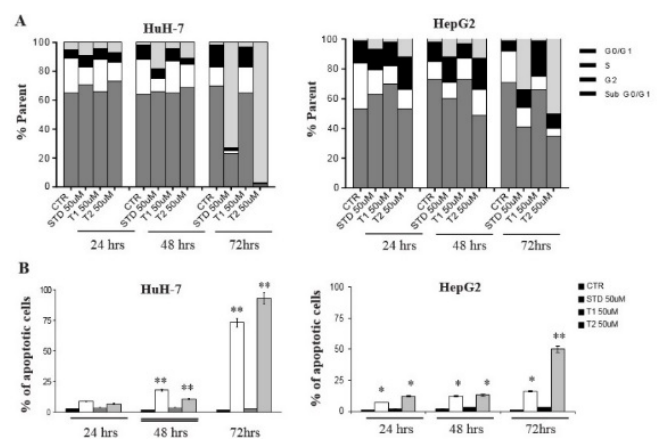

Figure 3: (A) Effect of STD, T1 and T2 turmeric powders $(50 \mu \mathrm{M})$ on Huh-7 and HepG2 cell cycle after 24, 48 and 72 hrs of treatment. The graphs show the percentage of cells in each cycle phase; (B) Percentage of Huh-7 and HepG2 apoptotic cells after 24, 48 and 72 hrs of treatment with STD, T1 and T2 turmeric powders $50 \mu \mathrm{M}$. * $\mathrm{p}<0.05 ;{ }^{* \star} \mathrm{p}<0.001$. CTR: control; STD: standard.

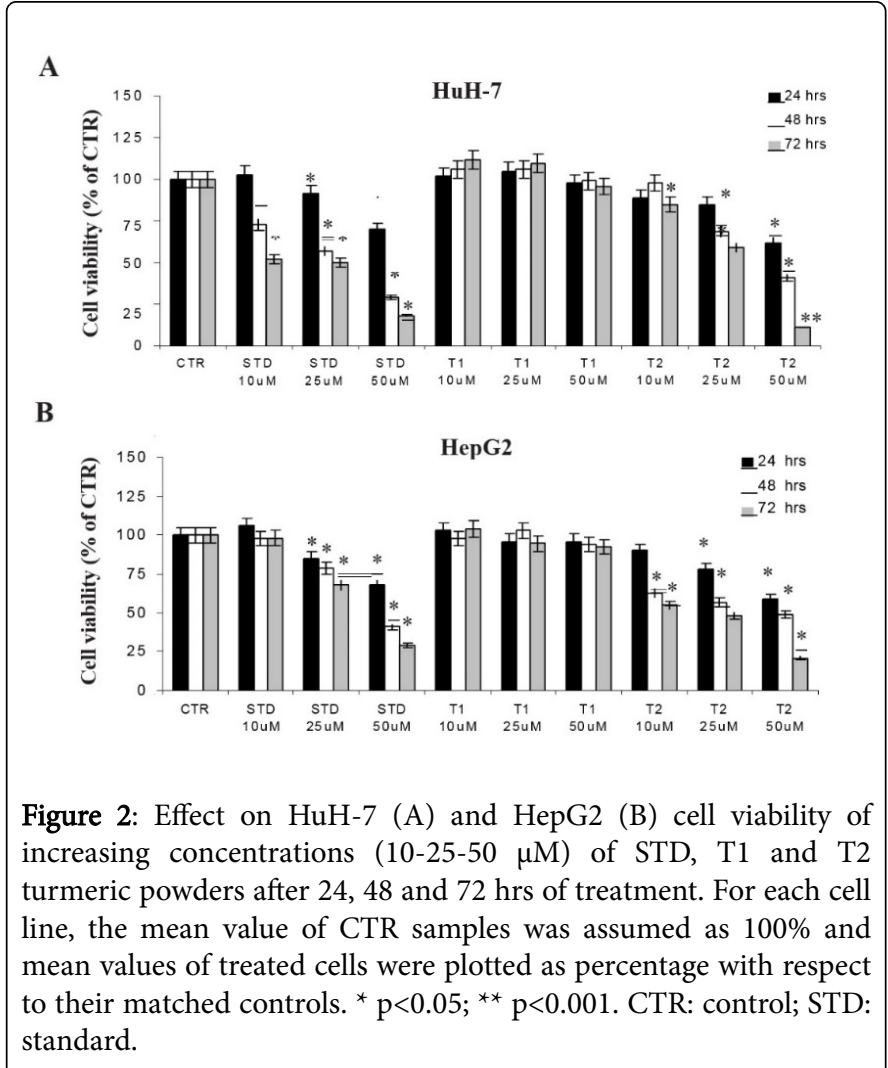

A
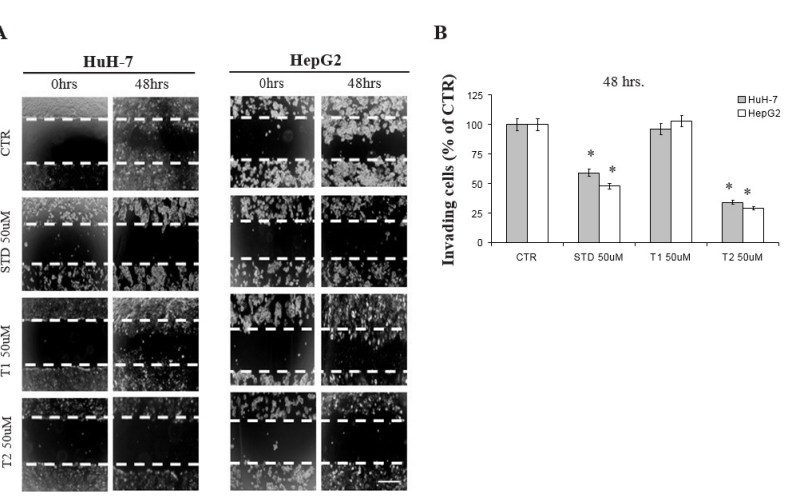

Figure 4: (A) Effect of STD, T1 and T2 turmeric powders $(50 \mu \mathrm{M})$ on Huh-7 and HepG2 cell migration at time 0 and after 48 hrs of treatment. Scale bar $(50 \mu \mathrm{m})$; (B) Evaluation of Huh-7 and HepG2 invasive potential after $48 \mathrm{hrs}$ of treatment with STD, T1 and T2 turmeric powders $50 \mu \mathrm{M}$. For each cell line, the mean value of CTR samples was assumed as $100 \%$ and mean values of treated cells were plotted as percentage with respect to their matched controls. * $\mathrm{p}<0.05 ;{ }^{* *} \mathrm{p}<0.001$. CTR: control; STD: standard. 


\section{T2, but not T1, inhibits migration and invasive potential of HepG2 and HuH-7 cell lines}

Finally, we evaluated the effect of $\mathrm{T} 1$ and $\mathrm{T} 2$ powders on HepG2 and HuH-7 cell migration and invasive potential. Scratch wound-healing assay revealed a strong ability of $\mathrm{T} 2$ powder $50 \mu \mathrm{M}$ to inhibit cell migration in both cell lines after 48 hrs of treatment; no significative changes were instead detectable after treatment with T1 powder (Figure 4A). Notably, the decrease of HepG2 and HuH-7 cell migration was found to be associated with a significant reduction of invasiveness in cells treated with T2, but not T1, powder (Figure 4B).

\section{Discussion}

The identification of the extraction method allowing the best recovery of bioactive curcuminoids represents an intense area of investigation in the field of phytomedicine. Indeed, the processing conditions play important roles in determining the quality and efficacy of the phytopharmaceutical product. In this context, it is of great interest to undertake studies investigating the relationship between the extraction method and the biological properties of curcuminoid extracts. In the present study we investigated the differences in curcuminoid content and antitumoral properties of two turmeric powders obtained according to ethyl acetate extraction and to a common Indian practice of boiling of rhizomes in water followed by dehydration at the sun. Results obtained in our study clearly showed that extraction with ethyl acetate resulted in a significant recovery of curcuminoids from turmeric when compared to the STD reference of curcuminoid mixture. On the contrary, curcuminoids recovery accordingly to common Indian practice was found to be negligible. These findings were in agreement with the results obtained in cell line functional studies. Indeed, while T2 powder (with a high content of curcuminoids) showed a strong antitumoral effects in HepG2 and HuH-7 cell lines both in terms of cell cytotoxicity and migration/ invasiveness inhibition, T1 powder (with a very low content of curcuminoids) was found to be ineffective against these two parameters. The different antitumoral efficacy of T1 and T2 powders could be ascribable to the different capability to extract curcuminoids in aqueous medium compared to aprotic polar solvents (as ethyl acetate). Indeed, as already reported, the efficiency of curcuminoid extraction in pure water is negligible, although it could be increased adding ethanol. Moreover, curcuminoids mainly exists in the keto-enol form in aqueous media, where solubility slightly increases but fast hydrolysis occurs [14]. Overall the present study shows that curcuminoid extraction in aqueous media (as occurs in Indian practice) does not represent the best choice for best recovery of bioactive curcuminoids. Conversely, conventional extraction with ethyl acetate should be preferred to achieve curcuminoid extracts with significant biological properties. This aspect should be taken into account when choosing the best product to be employed in prevention and treatment of human diseases, including cancer.

\section{Acknowledgements}

The authors thank the Center for Applied Biomedical research (C.R.B.A., Sant'Orsola-Malpighi Hospital, University of Bologna, Bologna, Italy) for providing the facilities for the present study.

\section{Funding}

This work was supported by grant from Fundamental Oriented Research (RFO 2016) to Prof. G. Brandi.

\section{Conflicts of Interest}

All the authors declare no conflict of interest.

\section{References}

1. Ravindran J, Prasad S, Aggarwal BB (2009) Curcumin and Cancer Cells: How Many Ways Can Curry Kill Tumor Cells Selectively? AAPS J 11: 495-510.

2. Basnet P, Skalko-Basnet N (2011) Curcumin: an anti-inflammatory molecule from a curry spice on the path to cancer treatment. Molecules 16: 4567-4598.

3. Wahlström B, Blennow G (1978) A study on the fate of curcumin in the rat. Acta Pharmacol Toxicol 43: 86-92.

4. Watke PS, Sachin BS, Patil AA, Mohato DM, Band TH, et al. (2011) Optimization of microwave, ultra-sonic and supercritical carbon dioxide assisted extraction techniques for curcumin from Curcuma longa. Sep Purif Technol 79: 50-55.

5. Xu G, Hao C, Tian S, Gao F, Sun W, et al. (2017) A method for the preparation of curcumin by ultrasonic-assisted ammonium sulfate/ ethanol aqueous two-phase extraction. J Chromatogr B Analyt Technol Biomed Life Sci 1041-1042: 167-174.

6. Kiamahalleh M (2016) High performance curcumin subcritical water extraction from turmeric (Curcuma longa L.). J Chromatogr B Analyt Technol Biomed Life Sci 1022: 191-198.

7. Kwon HL, Chung MS (2015) Pilot-scale subcritical solvent extraction of curcuminoids from Curcuma longa L. Food Chem 185: 58-64.

8. Sampathu SR, Krishnamurthy N, Sowbagya HB, Shankaranarayanan ML (1988) Studies on quality of (Curcuma longa) in relation to curing methods. J Food Sci Technol 25: 152-155.

9. Sahne F, Mohammadi M, Najafpour G, Moghadamnia A (2016) Extraction of bioactive compound curcumin from turmeric (Curcuma longa L.) via different routes: A comparative study. J Biotechnol 13: 173-180.

10. Yadav DK, Sharma K, Dutta A, Kundu A, Awasthi A, et al. (2017) Purity Evaluation of Curcuminoids in the Turmeric Extract Obtained by Accelerated Solvent Extraction. J AOAC 100: 586-591.

11. Syed HK, Liew KB, Loh GO, Peh KK (2015) Stability indicating HPLCUV method for detection of curcumin in Curcuma longa extract and emulsion formulation. Food Chem 170: 321-326.

12. Wichitnithad W, Jongaroonngamsang N, Pummangura S, Rojsitthisak P (2009) A simple isocratic HPLC method for the simultaneous determination of curcuminoids in commercial turmeric extracts. Phytochem Anal 20: 314-319.

13. Hasima N, Aggarwal BB (2012) Cancer-linked targets modulated by curcumin. Int J Biochem Mol Biol 3: 328-351.

14. D'Archivio AA, Maggi MA, Ruggieri F (2018) Extraction of curcuminoids by using ethyl lactate and its optimisation by response surface methodology. J Pharm Biomed Anal 149: 89-95. 